\title{
Intratumoral expression of mature human neutrophil peptide-1 potentiates the therapeutic effect of doxorubicin in a mouse 4T1 breast cancer model
}

\author{
DAN LI ${ }^{1 *}$, QING QIN $^{1 *}$, XIAO-YI WANG ${ }^{2 *}$, HUA-SHAN SHI ${ }^{1}$, MIN LUO $^{1}$, \\ FU-CHUN GUO $^{1}$, WEI WANG ${ }^{1}$ and YONG-SHENG WANG ${ }^{1}$ \\ ${ }^{1}$ Department of Thoracic Oncology, Cancer Center, State Key Laboratory of Biotherapy, West China Hospital, \\ Sichuan University, Chengdu, Sichuan; ${ }^{2}$ Department of Endocrine Surgery, The First Affiliated Hospital, \\ Chongqing Medical University, Chongqing, P.R. China
}

Received August 13, 2013; Accepted October 10, 2013

DOI: 10.3892/or.2013.2947

\begin{abstract}
Human neutrophil peptides (HNPs) 1-3 possess a high degree of similarity and are deregulated in many types of human tumors. Previous studies have demonstrated that tumor cell lines and microdissected fresh tumor cells express HNP1-3. In vitro, HNP1-3 have been reported to be cytotoxic to various types of tumor cells. Previously, we observed that the intracellular expression of HNP1 increased plasma membrane permeability in A549 cells and inhibited in vivo tumor angiogenesis. Based on these findings, we inferred that the intratumoral expression of HNP1 may benefit chemotherapy in solid tumors. In the present study, we established a mouse 4T1 breast cancer model imitating locally advanced breast cancer (LABC) and we injected the mice intratumorally with plasmid HNP1 (pHNP1). Doxorubicin (Dox) was administered twice i.v. at $5 \mathrm{mg} / \mathrm{kg}$ body weight on day 1 and 8 . The possible mechanisms were investigated by evaluating cell proliferation and apoptosis, intracellular Dox accumulation, mitochondrial transmembrane potential $\left(\Delta \Psi_{\mathrm{m}}\right)$ and ultrastructural alteration of intratumoral microvessels. Compared with the single agent treatment, the combination of Dox and pHNP1 resulted in a significant delay in in vivo tumor growth and a decrease in lung metastasis. This chemosensitization effect was also observed in an A549 lung cancer model treated with cisplatin (DDP) and pHNP1. MTT assay and Annexin V staining demonstrated that $4 \mathrm{~T} 1$ cells pre-transfected with pHNP1
\end{abstract}

Correspondence to: Professor Yong-Sheng Wang, Department of Thoracic Oncology, Cancer Center, State Key Laboratory of Biotherapy, West China Hospital, Sichuan University, Guo Xue Xiang No. 37, Chengdu, Sichuan, P.R. China

E-mail:wangys75@gmail.com

\section{*Contributed equally}

Key words: breast cancer, chemotherapy, doxorubicin, gene therapy, human neutrophil peptide-1 were significantly more sensitive to Dox, causing increased proliferation inhibition and apoptosis. Further investigations showed that the intracellular expression of HNP1 enhanced Dox accumulation and significantly impaired mitochondrial $\Delta \Psi_{\mathrm{m}}$. Moreover, in tumor tissues, HNP1 mediated intratumoral microvessel normalization and caused significant in situ tumor cell apoptosis. Our study suggests that intratumoral expression of HNP1 can significantly improve the therapeutic efficacy of Dox in breast cancer, abrogate the influence of multidrug resistance and enhance medication safety. Our findings may be important for the clinical therapeutic strategies of cancer chemotherapy.

\section{Introduction}

Locally advanced breast cancer (LABC) is the advanced stage of non-metastatic breast tumors and constitutes $\sim 10-20 \%$ of newly diagnosed breast cancers (1). Although the majority of breast cancer patients with early stage disease can be cured by surgery, LABC remains a clinical challenge. Despite appropriate therapy, the majority of patients with LABC develop distant metastasis and experience cancer relapse. The treatment of LABC requires a combination of chemotherapy, surgery and radiotherapy. Chemotherapy can effectively prevent the occurrence of tumor cell dissemination and metastasis (2). In particular, the administration of neoadjuvant chemotherapy is necessary for LABC patients; it can render inoperable tumors resectable and increases the rate of breast-conserving therapy (3).

Doxorubicin (Dox), one of the most effective anticancer chemotherapeutics, is commonly used in breast cancer chemotherapy. However, its clinical use is restricted by intrinsic or acquired multidrug resistance (MDR) and dose-dependent toxicity. As the major barrier to successful chemotherapy, MDR is often associated with the overexpression of ATP-binding cassette $(\mathrm{ABC})$ transporters in cancer cells. $\mathrm{ABC}$ transporters transduce the energy of ATP binding and hydrolysis into mechanical energy to translocate substrates across plasma membranes (4). By transporter-mediated drug efflux, cancer cells escape from chemotherapy caused injury. 
Human $\alpha$-defensins (HNP1-4) are small cationic antimicrobial peptides (CAPs) that are expressed mainly in neutrophils but also in specific subsets of T cells, monocytes and NK cells (5-7). Among them HNP1-3 possess a high degree of similarity in amino acid sequence and bioactivity. Deregulated expression and secretion of HNP1-3 have been detected in many types of human tumors (8-13), including lung and breast cancer $(12,13)$. Further investigations have found that tumor cell lines (8) and microdissected fresh tumor cells (10) express HNP1-3. At present, the influence of intratumorally expressed HNP1-3 on cancer therapy is unknown.

Studies have reported that HNP1-3 are cytotoxic to a variety of tumor cells (14-16). Previously, we observed that intracellularly expressed HNP1 increased plasma membrane permeability in human A549 lung carcinoma cells (17). We also noted that the intratumoral expression of HNP1 mediated specific angiogenesis inhibition in vivo $(17,18)$. In addition, HNP1 can cause VEGF-dependent endothelial cell proliferation inhibition by forming a ternary complex with fibronectin and $\alpha_{5} \beta_{1}$ integrin (19). In addition, there are reports that inhibition of VEGF-mediated angiogenesis can improve chemotherapy efficacy via inducing tumor microvessel normalization $(20,21)$. Based on these findings, we speculated that the intratumoral expression of HNP1 may play a beneficial role in cancer chemotherapy.

In the present study, we aimed to investigate the potential influence of intratumorally expressed HNP1 on breast cancer chemotherapy. We established a 4T1 tumor mouse model to imitate LABC, and we used treatment with combined therapy of HNP1 and Dox. The effect of the combination treatment was determined, and the possible mechanisms were probed. Our study suggests that intratumoral expression of HNP1 can significantly improve the therapeutic efficacy of Dox in breast cancer, abrogate the influence of multidrug resistance and enhance medication safety. HNP1 may function as a promising alternative to enhance the clinical chemotherapeutic response.

\section{Materials and methods}

Animals and tumor cell lines. Female BALB/c and female nude mice, 6-8 weeks of age, were purchased from the West China Experimental Animal Center (Chengdu, China). Mouse mammary carcinoma cell line 4T1 and human lung adenocarcinoma cell line A549 were purchased from ATCC and cultured in Dulbecco's modified Eagle's medium (DMEM), supplemented with $10 \%$ heat-inactivated fetal bovine serum (both from Invitrogen Life Technologies) and penicillin-streptomycin. The cell cultures were maintained in a humidified chamber at $37^{\circ} \mathrm{C}$ in $5 \% \mathrm{CO}_{2}$ atmosphere. The handling of mice and experimental procedures were conducted in accordance with protocols of the Ethics Committee of Sichuan University.

MTT cell viability assay. Cell viability was assessed by the MTT assay. Doxorubicin hydrochloride (Meiji Co.) was freshly prepared before each experiment. A recombinant plasmid pSecTag-HNP1 (pHNP1) that expresses a secretable form of mature HNP1 was previously constructed (17) and used in the present study. The concentration of MTT (Sigma) solution was $5 \mathrm{mg} / \mathrm{ml}$ dissolved in phosphate-buffered saline
(PBS). An untreated group, a Dox group, a pHNP1 group, and a pHNP1 plus Dox group were designed for this experiment. The 4T1 cells were seeded on 96-well plates at a density of $5 \times 10^{3}$ cells/well in $200 \mu 1$ DMEM medium and were allowed to adhere by incubation overnight. Then $0.2 \mu \mathrm{g}$ pHNP1 was transfected in the combined group and the pHNP1 group, using Lipofectamine 2000 reagent (Invitrogen Life Technologies). Transfection was conducted according to the manufacturer's instructions. Twelve hours later, different concentrations of Dox were added to the culture medium. After a 36-h incubation with Dox, MTT assay was conducted. The absorbance value of solution in each well was measured using a spectrophotometer (Thermo Scientific) at $570 \mathrm{~nm}$. Experiments were repeated 3 times. The absorbance value of the untreated cells was considered to be $100 \%$. The percentage of cell viability was determined by comparison with the untreated control.

Analysis of apoptosis. For the detection of apoptosis, 4T1 cells were seeded into 6-well plates at a density of $2 \times 10^{5}$, which were divided into a pHNP1 group, a Dox group, a pHNP1 plus Dox group and an untreated group. When cultured to $50 \%$ confluence, cells in the pHNP1 group and the combined group were transfected with $2 \mu \mathrm{g}$ pHNP1. Transfection was conducted as mentioned above. Twelve hours after transfection was carried out, Dox was added at a final concentration of $1 \mu \mathrm{g} / \mathrm{ml}$. Twenty-four hours later, both attached and floating cells were harvested and washed twice with PBS. After that, cells were resuspended and stained with the Annexin V-FITC kit (KeyGen). Apoptosis of cells was analyzed by a flow cytometer (BD Biosciences).

Detection of intracellular Dox accumulation. Intracellular Dox accumulation was detected according to Bruno and Slate (22). In this experiment, 4T1 cells were seeded into 6 -well plates at a density of $2 \times 10^{5}$ cells/well, which were divided into a Dox group, a pHNP1 plus Dox group and an untreated group. When cultured to $50 \%$ confluence, cells in the combined group were transfected with $2 \mu \mathrm{g}$ pHNP1 as mentioned above. Twelve hours after transfection was carried out, Dox was added at a final concentration of $1 \mu \mathrm{g} / \mathrm{ml}$. After $2 \mathrm{~h}$ of incubation in a humidified chamber, 4T1 cells from each well were harvested and washed twice with PBS. After that, cells were resuspended in $200 \mu 1$ PBS, and fluorescent intensity was detected by a flow cytometer.

Determination of mitochondrial transmembrane potential. For the detection of mitochondrial transmembrane potential $\left(\Delta \Psi_{\mathrm{m}}\right)$, Rhodamine 123 (Rh123; Sigma) staining was conducted. Briefly, 4T1 cells were seeded into 6-well plates at a density of $2 \times 10^{5}$, and were divided into a pHNP1 group, a Dox group, a pHNP1 plus Dox group and an untreated group. When cultured to $50 \%$ confluence, cells in the pHNP1 group and the combination group were transfected with $2 \mu \mathrm{g}$ pHNP1 as mentioned above. Twelve hours after transfection was conducted, Dox was added at a final concentration of $1 \mu \mathrm{g} /$ $\mathrm{ml}$. Twenty-four hours later, cells were harvested and washed twice with PBS. After that, cells were resuspended in $1 \mu \mathrm{g} / \mathrm{ml}$ Rh123 solution and incubated for $10 \mathrm{~min}$ at $37^{\circ} \mathrm{C}$. The stained cells were then washed twice and resuspended in $200 \mu \mathrm{l}$ PBS. Fluorescent intensity was detected by a flow cytometer. 

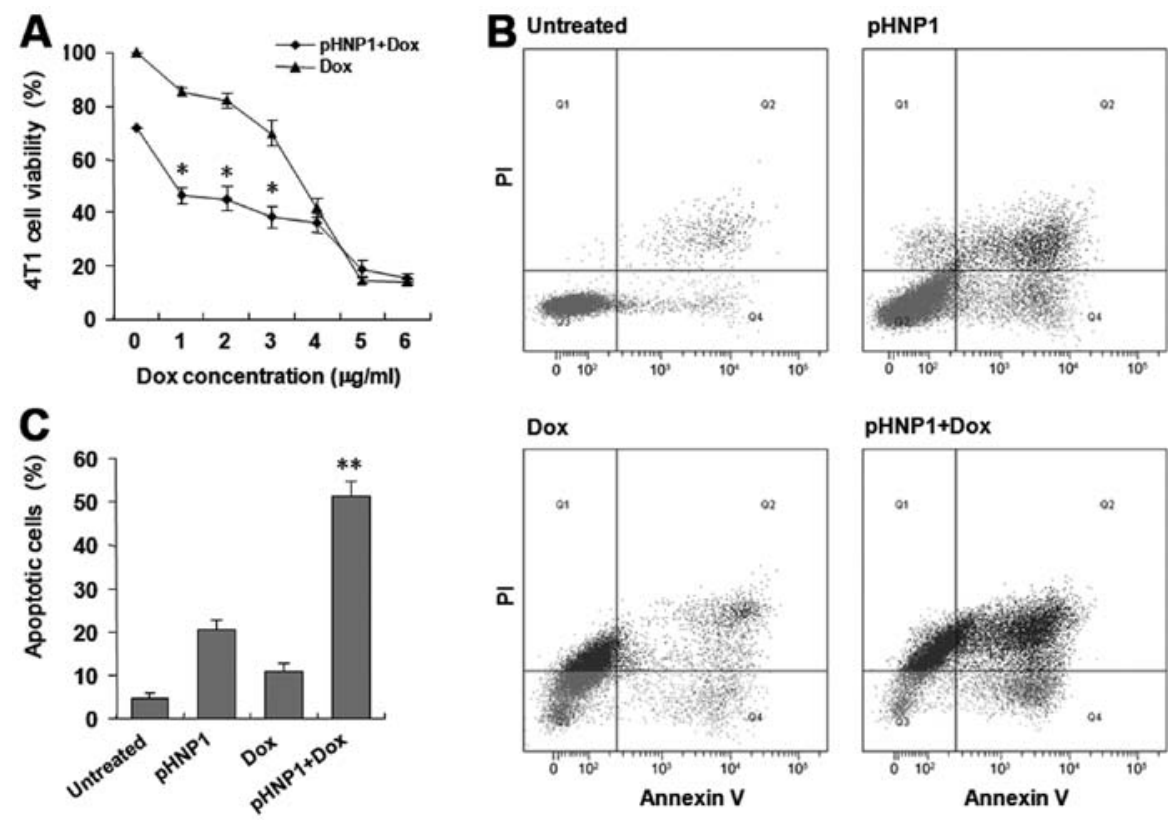

Figure 1. HNP1 significantly augments the chemosensitivity of 4T1 cells in vitro. (A) HNP1 significantly enhanced Dox-mediated inhibition of proliferation 4T1 cells were plated in 96-well plates in triplicate and transfected with pHNP1. Twelve hours after transfection was carried out, Dox was added at various concentrations $(0-6 \mu \mathrm{g} / \mathrm{ml})$. Thirty-six hours later, MTT assay was carried out. Data are representative of 3 independently conducted experiments and are shown as a percentage of the optical density value obtained from the untreated group ( $\mathrm{P}<0.05)$. (B and C) HNP1 significantly enhances Dox-mediated apoptosis. 4T1 cells were plated into 6-well plates in triplicate and transfected with pHNP1. Twelve hours later, a low dose of Dox (1 $\mu \mathrm{g} / \mathrm{ml}) \mathrm{was}$ added, and incubation was carried out for $24 \mathrm{~h}$. Annexin V-PI staining was conducted to detect cell apoptosis. Data are representative of 3 experiments performed and are shown as means $\pm \mathrm{SD}\left({ }^{* *} \mathrm{P}<0.01\right)$. HNP1, human neutrophil peptide-1; Dox, doxorubicin.

Tumor models and treatment. To detect the in vivo combination effect, 4T1 breast carcinoma and A549 lung adenocarcinoma models were established. For the $4 \mathrm{~T} 1$ model, $3 \times 10^{5} 4 \mathrm{~T} 1$ cells were injected subcutaneously into the right dorsal flank of $\mathrm{BALB} / \mathrm{c}$ mice. When the tumor diameter reached 7-8 mm, mice were randomly divided into a pHNP1 group, a Dox group, a pSec (empty vector) group, a combination group and a glucose (GS) group. Each group included 10 mice and each mouse was injected intratumorally and around the tumor with pHNP1 $(100 \mu \mathrm{g})$, pSec $(100 \mu \mathrm{g})$ or GS $(100 \mu \mathrm{l})$. The DNA was encapsulated in cationic liposome with a ratio of 1:3 and administered once every 3 days in a volume of $100 \mu$ for a total of 5 times. Dox was administered intravenously (i.v.) on day 1 and 8 at $5 \mathrm{mg} / \mathrm{kg}$ body weight. The control group received an injection of $100 \mu 15 \%$ glucose solution. For the A549 model, 5x10 6 A549 cells were implanted subcutaneously into the right dorsal flank of nude mice. When the tumor diameter reached $5 \mathrm{~mm}$, mice were randomly divided into 4 groups with 5 mice/group. DNA was administered as mentioned above. DDP was injected i.v. on day 1 and 8 at $5 \mathrm{mg} / \mathrm{kg}$ body weight. Tumor growth was evaluated by measurement of tumor diameters every 3 days and tumor volume was calculated as length $\mathrm{x}$ width ${ }^{2} \mathrm{x} 0.52$. For ethical reasons, experiments were terminated when tumor volume reached 2,000 $\mathrm{mm}^{3}$. Metastasis of the 4T1 tumors was evaluated by counting the lung metastatic nodules.

Tumor microvessel detection. To observe intratumoral microvessel change and avoid possible influence of the plasmid vector, 4T1 cells were injected subcutaneously into the right dorsal flank of BALB/c mice. When the tumor diameter reached 7-8 mm, a commercially mature HNP1 peptide (Abcam) was injected intratumorally once a day for a total of 3 times at a dose of $2 \mu \mathrm{g}$. Tumor tissues were harvested on day 4 , and the ultrastructure of the tumor microvessels was observed by transmission electron microscopy. For specimen preparation, tumor tissues were fixed with $2.5 \%$ glutaraldehyde in $0.1 \mathrm{~mol} / \mathrm{l} \mathrm{PBS}$ (pH 7.4) and then dehydrated in a graded series of acetone and embedded in Epon 812. Tumor microvessels were orientated and identified in semi-thin sections $(1 \mu \mathrm{m})$ that were stained with toluidine blue. Ultra-thin sections $(70 \mathrm{~nm})$ were poststained with uranyl acetate and lead citrate and examined under an $\mathrm{H}-7650$ electron microscope (Hitachi) at $80 \mathrm{kV}$. Images were captured by electron microscopy and analyzed.

TUNEL assay. For in vivo apoptosis detection, tumor tissues were removed from the tumor-bearing mice $48 \mathrm{~h}$ after the last treatment and fixed with freshly prepared $4 \%$ paraformaldehyde in PBS (pH 7.4). After fixation, tumor tissues were dehydrated and embedded into paraffin. Paraffin sections $(4 \mu \mathrm{m})$ were constructed by a paraffin slicing machine (Leica, RM2235). Staining was performed using the protocol supplied with the Promega terminal deoxynucleotidyl-transferasemediated dUTP nick end labeling (TUNEL) assay kit.

Statistical analysis. SPSS 11.5 was used for statistical analysis. The statistical significance of results in all experiments was determined by Student's t-test and ANOVA. The findings were regarded as significant at $\mathrm{P}<0.05$.

\section{Results}

HNP1 potentiates Dox-mediated $4 T 1$ cell proliferation inhibition and apoptosis in vitro. To determine the influence of HNP1 on Dox-mediated cell growth inhibition and apoptosis, 
MTT assay and Annexin V-FITC staining were conducted. To avoid the possible influence of chemotherapeutics on HNP1 expression, Dox was added $12 \mathrm{~h}$ after transfection was carried out. For detection of proliferation, in the pHNP1 group and the pHNP1 plus Dox group, 4T1 cells were transfected with pHNP1. Then Dox was added to the cells in the Dox group and the pHNP1 plus Dox group at gradient concentrations $(0-6 \mu \mathrm{g} / \mathrm{ml})$. After $36 \mathrm{~h}$ of incubation with Dox, MTT assay was carried out. The 4T1 cells treated with Dox alone showed dose-dependent growth inhibition, and the concentration showing $50 \%$ growth inhibition in culture $\left(\mathrm{IC}_{50}\right)$ was $\sim 3.5 \mu \mathrm{g} / \mathrm{ml}$. Following the combined treatment, this concentration decreased to $1 \mu \mathrm{g} / \mathrm{ml}$ (Fig. 1A).

The quantitative assessment of apoptosis was conducted by PI and Annexin V-FITC double staining and was evaluated by flow cytometry (Fig. 1B). 4T1 cells were transfected with pHNP1 and Dox was added at a concentration of $1 \mu \mathrm{g} /$ $\mathrm{ml}$. Twenty-four hours later, both attached and floating cells were harvested and stained. Analysis showed that 4T1 cells treated with the combination of pHNP1 and Dox (51.5\%) had a significantly increased apoptosis rate $(\mathrm{Q} 2+\mathrm{Q} 4)$ when compared with the rate of cells treated with pHNP1 (20.6\%) or Dox (11.1\%) alone (Fig. 1C). The apoptotic rate following combination treatment was greater than the sum of the rates following each treatment alone, suggesting that the usage of pHNP1 and Dox together had a synergistic effect.

HNP1 enhances intracellular Dox accumulation in $4 T 1$ cells. Dox has autofluorescent properties that enable the evaluation of drug accumulation by flow cytometry. The intracellular concentration of chemotherapeutics is closely related to the therapeutic effect. To explore the possible mechanism of HNP1-mediated cytotoxicity enhancement, 4T1 cells were

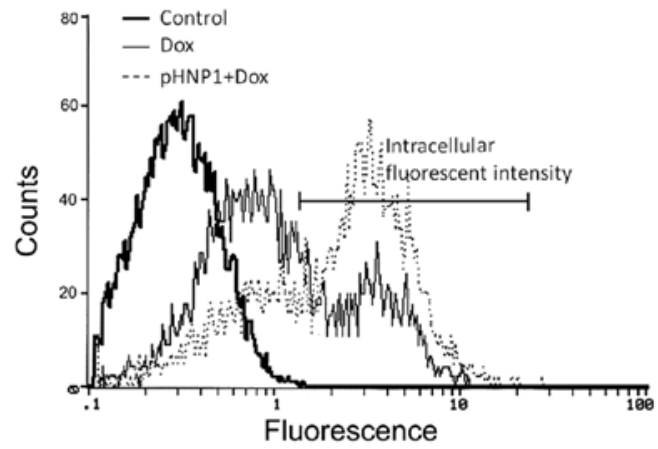

Figure 2. HNP1 increases the intracellular Dox accumulation in 4T1 cells 4T1 cells treated with a combination of HNP1 and Dox showed obviously increased intracellular fluorescent intensity $(72.3 \%)$ when compared to that in cells treated with Dox alone $(42.4 \%)$. 4T1 cells were plated into 6-well plates and transfected with pHNP1. Twelve hours after transfection was conducted, Dox $(1 \mu \mathrm{g} / \mathrm{ml})$ was added, and incubation was carried out for $2 \mathrm{~h}$ in a humidified chamber. Cells from each group were harvested and analyzed for Dox-specific fluorescence by flow cytometry. Untreated 4T1 cells served as an empty control. HNP1, human neutrophil peptide-1; Dox, doxorubicin.

treated with Dox and detected for intracellular fluorescent intensity. In this experiment, $12 \mathrm{~h}$ after pHNP1 transfection, Dox was added at a concentration of $1 \mu \mathrm{g} / \mathrm{ml}$ and incubated for $2 \mathrm{~h}$. Flow cytometric analysis showed that, compared with the Dox group, 4T1 cells in the pHNP1 plus Dox group exhibited an increase in both the percentage of cells with fluorescence and intracellular fluorescent intensity (Fig. 2). This finding indicated that the treatment of pHNP1 caused an enhancement of intracellular accumulated Dox in 4T1 cells.

HNP1 causes dissipation of mitochondrial transmembrane potential in $4 T 1$ cells. The internalization of HNP1-3 may

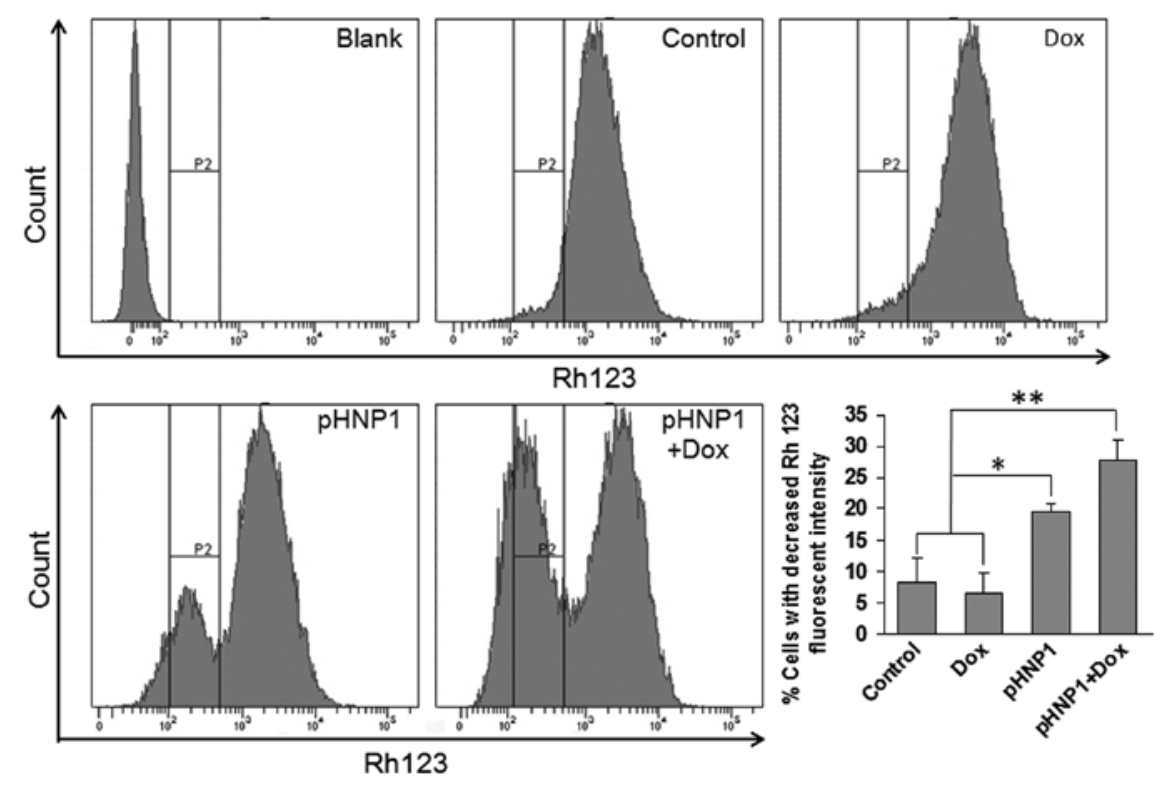

Figure 3. HNP1 significantly decreases the mitochondrial transmembrane potential $\left(\Delta \Psi_{\mathrm{m}}\right)$ of $4 \mathrm{~T} 1$ cells. Loss of mitochondrial integrity in $4 \mathrm{~T} 1$ cells was detected by Rh123 staining and is shown as the percentage of reduced Rh123 fluorescent intensity (P2). The blank group refers to the untreated and unstained 4T1 cells. The control group refers to 4T1 cells which were not treated with either HNP1 or Dox, but stained with Rh123. As shown, expression of HNP1 significantly induced $\Delta \Psi_{\mathrm{m}}$ decrease in $4 \mathrm{~T} 1$ cells. The combined treatment of HNP1 and Dox caused a further decrease. Dox alone exhibited no significant influence on $\Delta \Psi_{\mathrm{m}}$. In this experiment, 4T1 cells were seeded into 6-well plates in triplicates and transfected with pHNP1 as described in Materials and methods. Dox $(1 \mu \mathrm{g} / \mathrm{ml})$ was added, and incubation was carried out for $24 \mathrm{~h}$. Data are representative of 3 independent experiments performed and are shown as means \pm SD ( $\left.\mathrm{P}<0.05,{ }^{* *} \mathrm{P}<0.01\right)$. HNP1, human neutrophil peptide-1; Rh123, Rhodamine 123; Dox, doxorubicin. 

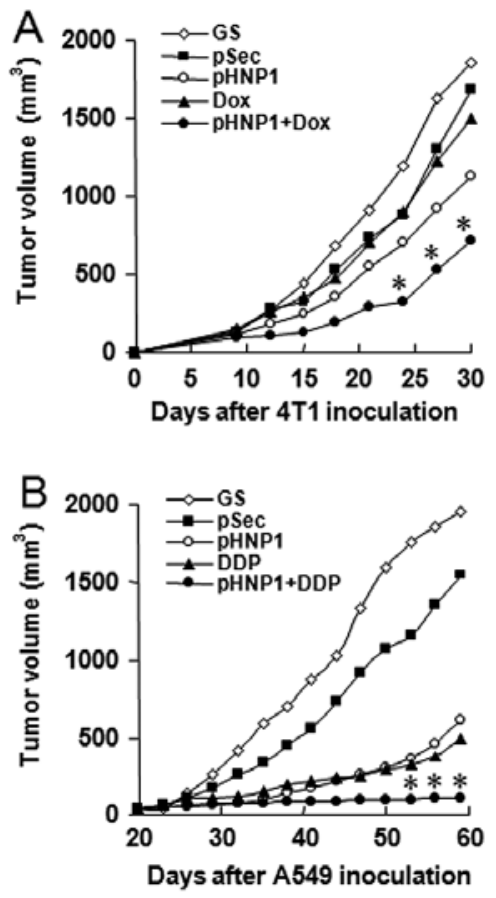

Figure 4. HNP1 significantly augments the chemosensitivity of chemotherapeutic drugs in vivo. (A) Intratumoral expression of HNP1 significantly augmented the therapeutic efficacy of Dox in the s.c. 4T1 cell tumor model. When the tumor volume reached $100 \mathrm{~mm}^{3}$, 4T1 tumor-bearing mice were treated with an intratumoral injection of pHNP1 $(100 \mu \mathrm{g})$ every 3 days for a total of 5 times. Dox was administered i.v. on day 1 and 8 at a dose of $5 \mathrm{mg} / \mathrm{kg}$ body weight. There were 10 mice in each group. (B) Intratumoral expression of HNP1 significantly augmented the therapeutic efficacy of DDP in the s.c. A549 cell tumor model. When the tumor volume reached $50 \mathrm{~mm}^{3}$, A549 tumor-bearing mice were treated with an intratumoral injection of pHNP1 $(100 \mu \mathrm{g})$ every 3 days for a total of 5 times. DDP was administered i.v. on day 1 and 8 at a dose of $5 \mathrm{mg} / \mathrm{kg}$ body weight. There were 5 mice in each group. Data are shown as means $\pm \mathrm{SD}\left({ }^{*} \mathrm{P}<0.05\right)$. HNP1, human neutrophil peptide-1; Dox, doxorubicin.

disrupt the membrane integrity of cancer cell mitochondria (23). We therefore employed the fluorescent dye Rh123, which localizes to intact mitochondria, to determine the effect of HNP1 on mitochondrial membrane integrity. Fig. 3 shows that 4T1 cells in the pHNP1 group exhibited a significant decrease in Rh123 fluorescent intensity (P2). The combination treatment of pHNP1 and Dox caused a further decrease. In contrast, Dox had no significant influence on $\Delta \Psi_{\mathrm{m}}$. Our findings indicate that pHNP1 treatment results in the loss of mitochondrial membrane integrity and dissipation of $\Delta \Psi_{\mathrm{m}}$.

HNPI augments the chemosensitivity of Dox and DDP in vivo. To further explore the in vivo effect of the combination therapy, we established 4T1 breast carcinoma and A549 human lung adenocarcinoma models. 4T1 breast cancer cells have high metastatic potential. We and others found that apparent tumor metastasis could be monitored in BALB/c mice when the tumor diameter reached $10 \mathrm{~mm}$ (24). To imitate the therapy of LABC, we inoculated 4T1 cells into the right dorsal flank of $\mathrm{BALB} / \mathrm{c}$ mice and began treatment when the tumor diameter reached 7-8 $\mathrm{mm}$. A low dose of Dox $(5 \mathrm{mg} / \mathrm{kg}$ body weight) was administered i.v. on day 1 and 8. In the A549 model, treatment was started when the tumor diameter was $5 \mathrm{~mm}$. pHNP1 and DDP were administered at the same dose and frequency
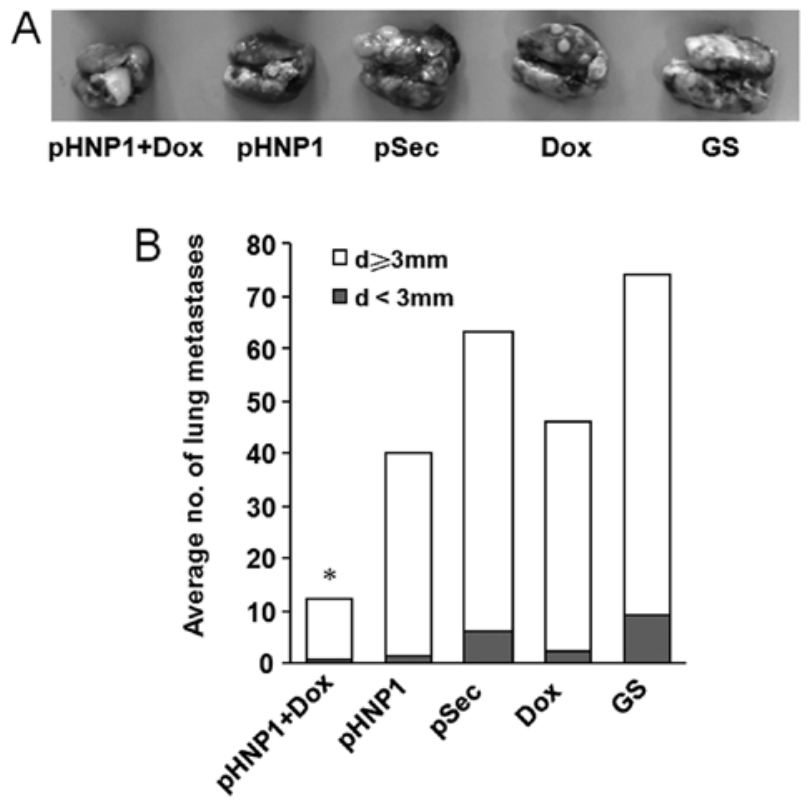

Figure 5. Anti-metastatic effect of the combined treatment of HNP1 and Dox in the 4T1 tumor model. Combination treatment of HNP1 plus Dox significantly impaired lung metastases of $4 \mathrm{~T} 1$ cell tumors. When tumor volumes reached $2,000 \mathrm{~mm}^{3}$, all 4T1-bearing mice were sacrificed, and lungs were harvested for measurement and counting of metastatic nodules. (A) Representative images of the lungs from tumor-bearing mice of each group. (B) Mean value of the lung metastatic nodules in each group. Column, mean number of nodules $\left({ }^{*} \mathrm{P}<0.01\right)$. Dox, doxorubicin.

as in the 4T1 model. Treatment with a combination of pHNP1 significantly augmented the therapeutic effect of chemotherapeutics in the 4T1 (Fig. 4A) and A549 (Fig. 4B) models. These results indicate that HNP1 enhanced the antitumor activity of the chemotherapy drugs in vivo. In both of the experiments, no obvious side-effect of the combination therapy was observed.

Combined treatment inhibits the metastasis of $4 T 1$ breast cancer. 4T1 breast cancer cells possess high potential for metastasis and can metastasize to several organs, particularly the lung. Lung metastasis of $4 \mathrm{~T} 1$ cells in BALB/c mice occurs at a high frequency and can better represent the extent of tumor spreading (24). For these considerations, when the 4T1 cell tumor volume reached $2,000 \mathrm{~mm}^{3}$, mice were sacrificed, and lung metastasis was examined under a dissecting microscope. The pHNP1 and Dox showed a similar effect of metastasis inhibition. However, mice that received a combined treatment of Dox and pHNP1 had significantly less lung metastatic nodes. Representative images of lungs are shown in Fig. 5A. The results of the analysis are expressed as the average number of metastatic nodules (Fig. 5B).

Intratumoral expression of HNP1 promotes tumor microvessel normalization. Tumor vasculature is characteristically disorganized with unstructured morphology, resulting in increased interstitial pressure and poor perfusion in tumors (25). This vasculature within tumors becomes a key obstacle for efficient drug delivery $(26,27)$. VEGF signaling plays an important role in tumor angiogenesis. Inhibition of VEGF or its kinase receptors (VEGFRs) could result in tumor vasculature normalization $(20,21)$. It has been reported that HNP1 impairs 


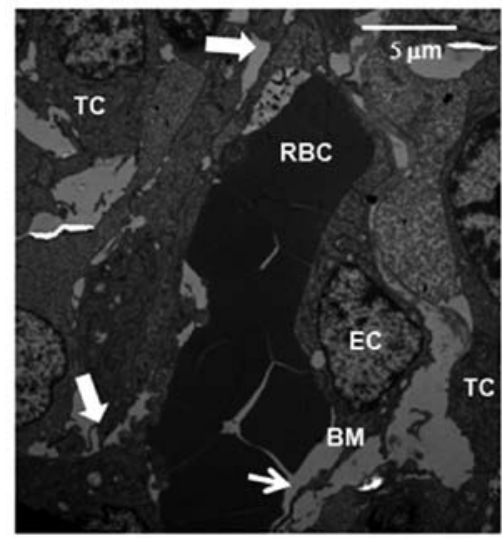

Untreated

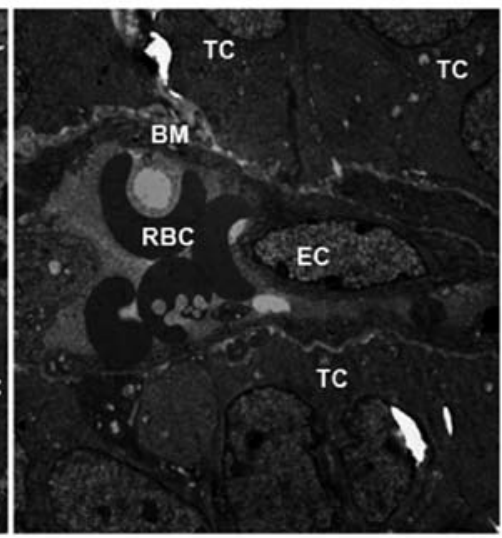

HNP1

Figure 6. Intratumoral expression of HNP1 promotes tumor microvessel normalization. Ultrastructural changes in tumor microvessels were observed by transmission electron microscopy. In the untreated group, tumor microvessels showed obvious structural abnormalities. EC sprouting (broad arrows), discontinuous vascular BM (thick arrows) and irregular lumen of blood vessel were observed. In contrast, in the HNP1-treated group, tumor microvessels exhibited significant structure normalization. EC, endothelial cell; BM, basement membrane; TC, tumor cell; RBC, red blood cell. Scale bar, $5 \mu$ m. HNP1, human neutrophil peptide-1.
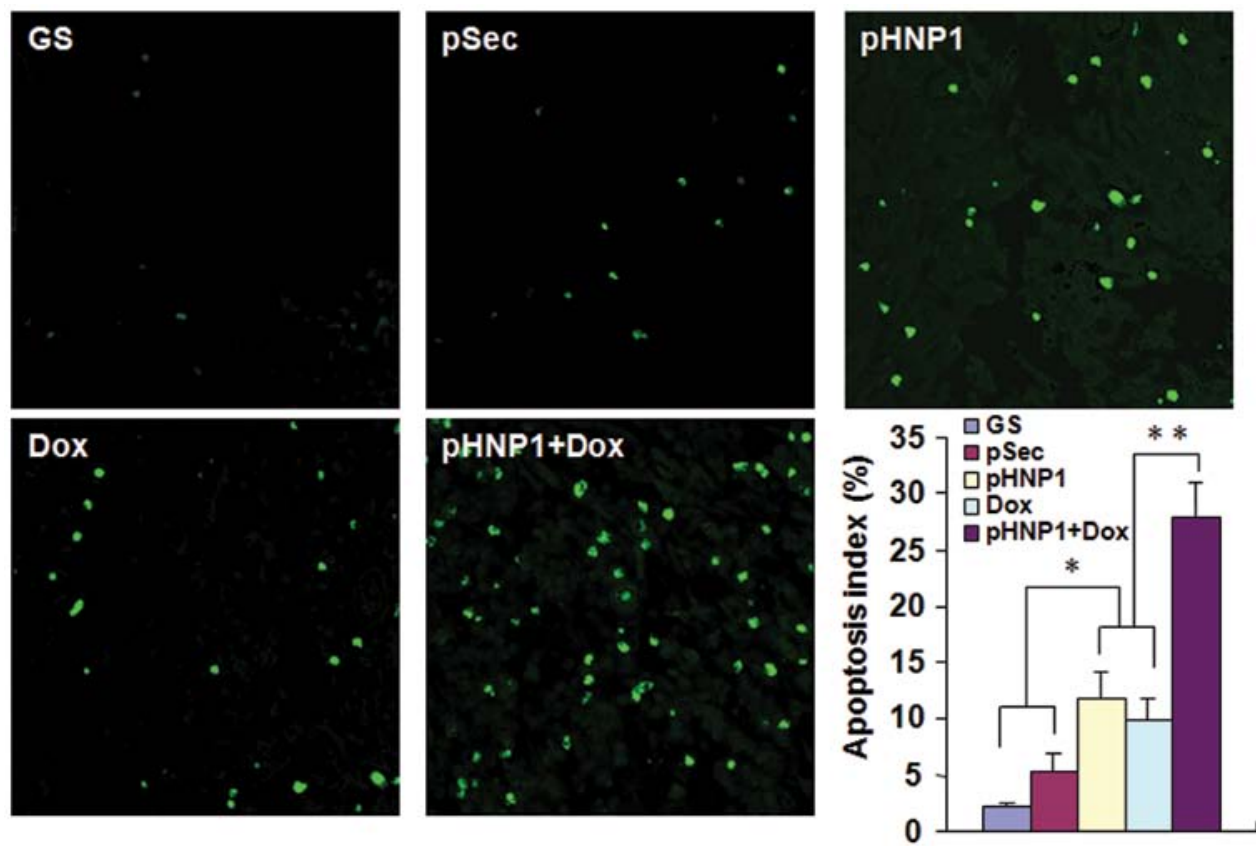

Figure 7. Combination treatment of HNP1 and Dox results in significantly increased tumor cell apoptosis in vivo. Tumor tissues were removed $48 \mathrm{~h}$ after the last treatment and TUNEL staining was carried out. The percentage of apoptosis was determined by counting the number of apoptotic cells (cells with green fluorescence) and dividing by the total number of cells in the field (5 high power fields/slide). Columns, mean; bars, \pm SD. ("P<0.05, " $\left.{ }^{* *}<0.01\right)$. HNP1, human neutrophil peptide-1; Dox, doxorubicin.

VEGF-mediated angiogenesis $(19,28)$. To detect the influence of HNP1 on tumor microvessels, a commercial HNP1 peptide was injected intratumorally, and ultrastructural changes were observed by transmission electron microscopy. The untreated tumors exhibited an obvious structural abnormality, accompanied by strong activity of vascular endothelium sprouting, discontinuous vascular basement membrane (BM) and irregular lumen of blood vessels (Fig. 6). In contrast, in the HNP1-treated group, tumor vessels were lined by an orderly formed single endothelial cell (EC) monolayer, which was continuous and tightly packed, with a normal polarity and intact BM (Fig. 6). Our observations suggest that the intra- tumoral expression of HNP1 promotes the normalization of tumor microvessels.

Combined treatment mediates increased tumor cell apoptosis in vivo. To evaluate the in vivo apoptosis of tumor cells, tumor tissues were removed $48 \mathrm{~h}$ after the last treatment, and in situ TUNEL staining was conducted. The TUNEL assay showed that the combination treatment resulted in a significantly higher number of apoptotic cells when compared to the number following the single treatment (Fig. 7). Similar results were also observed in the A549 model (data not shown). 


\section{Discussion}

CAPs are a class of natural-source cationic peptides and important components of the innate immune system $(29,30)$. In vitro, CAPs exhibit selective cytotoxicity against a broad spectrum of human cancer cells $(14,31-37)$ and this cytotoxic effect was equivalent when tested against sensitive and MDR cell lines (32,35-37). It was also reported that certain CAPs enhance the tumor killing activity of chemotherapeutic agents $(34,35,37)$. In the treatment of leukemia cells, cecropin A showed a synergistic effect when combined with S-Fu and ara-c (34). In another study of MDR tumor cells, mammalian-derived extended-helical cationic peptides and insect-derived $\alpha$-helical peptides significantly augmented the in vitro activity of Dox (35). Despite these findings, little is known about the in vivo influence of CAPs on chemotherapy and the possible mechanisms involved.

As members of the CAP family, HNP1-3 are highly similar in regards to bioactivities and are overexpressed in a variety of human tumors (8-13). In the present study, we showed for the first time that intratumorally expressed HNP1 augments the chemosensitivity of Dox in mouse 4T1 breast cancer. This chemosensitization effect was also noted in a human A549 lung cancer model treated with pHNP1 and DDP. Further investigations showed that the possible mechanisms involved significantly increased cancer cell apoptosis, augmented intracellular drug accumulation, decreased mitochondrial transmembrane potential $\left(\Delta \Psi_{\mathrm{m}}\right)$, impaired tumor metastasis and intratumoral vasculature normalization. We suggest that our findings may be important for clinical chemotherapeutic strategies.

$\mathrm{LABC}$ is the advanced stage of non-metastatic breast cancer. Even following proper treatment, most LABC patients still develop distant metastasis and experience cancer relapse. The clinical treatment of LABC requires a combination of therapeutic strategies, in particular, the administration of chemotherapeutics. Chemotherapy can prevent the occurrence of tumor cell dissemination and metastasis (2). In particular, the application of neoadjuvant chemotherapy can effectively reduce tumor burden and greatly enhance the rate of breast-conserving therapy of LABC patients (3). As one of the most effective chemotherapeutic drugs, Dox is commonly used against breast cancer; however, its clinical use is impeded by dose-dependent toxicity and MDR. 4T1 breast cancer cells have a high metastatic potential. In BALB/c mice, apparent tumor metastasis is monitored when tumor diameter is $\sim 10 \mathrm{~mm}$ (24). In the present study, we established a 4T1 tumor model to imitate $\mathrm{LABC}$, and treatment began when the tumor diameter reached $7-8 \mathrm{~mm}$. The clinically relevant dose of Dox was $10 \mathrm{mg} / \mathrm{kg}\left(30 \mathrm{mg} / \mathrm{m}^{2}\right)$. In this experiment, mice were administered i.v. a lower dose of $5 \mathrm{mg} / \mathrm{kg}$ weight twice on day 1 and 8 . We found that HNP1 significantly augmented the therapeutic effect of Dox and impaired tumor metastasis, accompanied by no obvious side-effects. This combination therapeutic strategy exhibited a more beneficial effect than each single treatment alone and decreased chemotherapeutic consumption. We suggest that HNP1 may provide a promising alternative to overcome the systemic side-effect of chemotherapeutics and improve medication safety.

There are many reasons that contribute to cancer cell survival during chemotherapy: increased drug efflux, enhanced repair/increased tolerance to DNA damage, high anti-apoptotic potential and decreased permeability. MDR is one of the major causes of chemotherapy failure in the clinical treatment of breast cancer, and is closely related with overexpression of $\mathrm{ABC}$ transporters (38) such as BCRP/ ABCG2 (39). ABC transporters confer a drug-resistant phenotype by decreasing intracellular accumulation of chemotherapeutic drugs via ATP-dependent efflux mechanisms (40). Previously, we found that HNP1 increased plasma membrane permeability in A549 cells (17). In the present research, the intracellular expression of HNP1 caused enhancement of Dox accumulation in 4T1 cells and exhibited a synergistic apoptosis-inducing effect in combination therapy. We also showed that HNP1 mediated the dissipation of $\Delta \Psi_{\mathrm{m}}$ in $4 \mathrm{~T} 1$ cells. It is reported that deprivation of cellular energy supply is an effective way to overcome MDR (41). The collapse of mitochondrial $\Delta \Psi_{\mathrm{m}}$ causes an uncoupling of the respiratory chain, and in this regard, HNP1 could greatly impair the energy source of $\mathrm{ABC}$ transporters and consequently breakdown of MDR. Based on these findings, we conclude that HNP1 enhances chemosensitivity by increasing the entrance of chemotherapeutics and at the same time decreasing drug efflux. Additionally, a previous study found that overexpression of anti-apoptotic protein $\mathrm{Bcl}-2 \mathrm{did}$ not affect HNP1-induced cell death in A549 cells (23). In addition, drastic membrane disruption function by HNP1 makes it difficult for cancer cells to develop resistance. Therefore, the administration of HNP1 is not easily influenced by apoptosis evasion or causes drug resistance. Our findings indicated that HNP1 may function as an effective chemosensitizer to overcome chemotherapy failure.

Modulation of intratumoral vasculature has been postulated as a method for improving drug delivery to solid tumors (42). Tumor vessels are tortuous and dilated, lack pericyte coverage and exhibit an abnormal basement membrane (43-45). Vessel normalization within tumors could result in increased drug perfusion and a higher sensitivity to standard chemotherapy and immunotherapy $(46,47)$. VEGF signaling plays an important role in tumor angiogenesis. Inhibition of VEGF or its kinase receptors (VEGFRs) can cause normalization of tumor vasculature $(20,21)$. It is reported that HNP1 could impair VEGF-mediated angiogenesis $(19,28)$. In the present study, to avoid the influence of the plasmid vector on angiogenesis, a mature HNP1 peptide was injected intratumorally in the 4T1 tumors and normalized microvessels of orderly formed EC monolayer were observed after treatment. Our findings revealed that the intratumorally expressed HNP1 also potentiated chemotherapy by inducing vasculature normalization in $4 \mathrm{~T} 1$ tumor tissues.

In our previous investigations, intratumorally expressed HNP1 exhibited multiple antitumor effects in vivo, and in particular, mediated host immune responses to tumors (18). Considering the impact of tumor burden on the potential efficacy of immunotherapy, in animal experiments, pHNP1 was administered when the tumor diameter reached $4 \mathrm{~mm}$. In the present research, to imitate LABC in a 4T1 tumor model and better investigate the relationship of HNP1 and chemotherapy, treatment started when the tumor diameter reached $\sim 7-8 \mathrm{~mm}$. As shown in Fig. 5, a single treatment of Dox $(5 \mathrm{mg} / \mathrm{kg}$ body weight) did not effectively inhibit tumor growth. In contrast, 
the combined therapy significantly delayed 4T1 tumor growth early in treatment when compared with the other groups. This chemosensitization effect was similar in the A549 model which was established in nude mice injected with DDP. However, the combination therapeutic effect did not reach significance under early intervention as in the 4T1 model. We conclude that an HNP-mediated host immune response may not play the major role in its chemosensitization function.

In summary, our research provides evidence that the expression of HNP1 in tumors may play a beneficial role in chemotherapy. Gene therapy based on HNP1 may provide an attractive alternative for cancer chemosensitization.

\section{Acknowledgements}

This study was supported by the National Major Project 2011ZX09302-001-01, and by the National Natural Science Foundation (NSFC81272523).

\section{References}

1. Franceschini G, Terribile D, Magno S, et al: Update in the treatment of locally advanced breast cancer: a multidisciplinary approach. Eur Rev Med Pharmacol Sci 11: 283-289, 2007.

2. Berry DA, Cronin KA, Plevritis SK, et al: Effect of screening and adjuvant therapy on mortality from breast cancer. N Engl J Med 353: 1784-1792, 2005

3. Schott AF and Hayes DF: Defining the benefits of neoadjuvant chemotherapy for breast cancer. J Clin Oncol 30: 1747-1749, 2012.

4. Dong J, Yang G and McHaourab HS: Structural basis of energy transduction in the transport cycle of MsbA. Science 308: 1023-1028, 2005.

5. Agerberth B, Charo J, Werr J, et al: The human antimicrobial and chemotactic peptides LL-37 and $\alpha$-defensins are expressed by specific lymphocyte and monocyte populations. Blood 96 3086-3093, 2000.

6. Obata-Onai A, Hashimoto S, Onai N, et al: Comprehensive gene expression analysis of human NK cells and $\mathrm{CD}^{+} \mathrm{T}$ lymphocytes. Int Immunol 14: 1085-1098, 2002.

7. Ganz T, Selsted ME and Lehrer RI: Defensins. Eur J Haematol 44: $1-8,1990$

8. Müller CA, Markovic-Lipkovski J, Klatt T, et al: Human $\alpha$-defensins HNPs-1, -2 , and -3 in renal cell carcinoma: influences on tumor cell proliferation. Am J Pathol 160: 1311-1324, 2002.

9. Lundy FT, Orr DF, Gallagher JR, et al: Identification and overexpression of human neutrophil $\alpha$-defensins (human neutrophil peptides 1,2 and 3) in squamous cell carcinomas of the human tongue. Oral Oncol 40: 139-144, 2004.

10. Melle C, Ernst G, Schimmel B, et al: Discovery and identification of $\alpha$-defensins as low abundant, tumor-derived serum markers in colorectal cancer. Gastroenterology 129: 66-73, 2005.

11. Holterman DA, Diaz JI, Blackmore PF, et al: Overexpression of $\alpha$-defensin is associated with bladder cancer invasiveness. Urol Oncol 24: 97-108, 2006

12. Bateman A, Singh A, Jothy S, Fraser R, Esch F and Solomon S: The levels and biologic action of the human neutrophil granule peptide HP-1 in lung tumors. Peptides 13: 133-139, 1992.

13. Li J, Zhao J, Yu X, et al: Identification of biomarkers for breast cancer in nipple aspiration and ductal lavage fluid. Clin Cancer Res 11: 8312-8320, 2005

14. Lichtenstein A, Ganz T, Selsted ME and Lehrer RI: In vitro tumor cell cytolysis mediated by peptide defensins of human and rabbit granulocytes. Blood 68: 1407-1410, 1986.

15. Barker E and Reisfeld RA: A mechanism for neutrophil-mediated lysis of human neuroblastoma cells. Cancer Res 53: 362-367, 1993.

16. McKeown ST, Lundy FT, Nelson J, et al: The cytotoxic effects of human neutrophil peptide-1 (HNP1) and lactoferrin on oral squamous cell carcinoma (OSCC) in vitro. Oral Oncol 42: 685-690, 2006.
17. Xu N, Wang YS, Pan WB, et al: Human $\alpha$-defensin-1 inhibits growth of human lung adenocarcinoma xenograft in nude mice. Mol Cancer Ther 7: 1588-1597, 2008.

18. Wang YS, Li D, Shi HS, et al: Intratumoral expression of mature human neutrophil peptide-1 mediates antitumor immunity in mice. Clin Cancer Res 15: 6901-6911, 2009.

19. Chavakis T, Cines DB, Rhee JS, et al: Regulation of neovascularization by human neutrophil peptides ( $\alpha$-defensins): a link between inflammation and angiogenesis. FASEB J 18: 1306-1308, 2004.

20. Shen G, Li Y, Du T, et al: SKLB1002, a novel inhibitor of VEGF receptor 2 signaling, induces vascular normalization to improve systemically administered chemotherapy efficacy. Neoplasma 59: 486-493, 2012.

21. Dickson PV, Hamner JB, Sims TL, et al: Bevacizumab-induced transient remodeling of the vasculature in neuroblastoma xenografts results in improved delivery and efficacy of systemically administered chemotherapy. Clin Cancer Res 13: 3942-3950, 2007.

22. Bruno NA and Slate DL: Effect of exposure to calcium entry blockers on doxorubicin accumulation and cytotoxicity in multidrug-resistant cells. J Natl Cancer Inst 82: 419-424, 1990.

23. Aarbiou J, Tjabringa GS, Verhoosel RM, et al: Mechanisms of cell death induced by the neutrophil antimicrobial peptides $\alpha$-defensins and LL-37. Inflamm Res 55: 119-127, 2006.

24. Pulaski BA, Terman DS, Khan S, Muller E and OstrandRosenberg S: Cooperativity of Staphylococcal aureus enterotoxin B superantigen, major histocompatibility complex class II, and CD80 for immunotherapy of advanced spontaneous metastases in a clinically relevant postoperative mouse breast cancer model. Cancer Res 60: 2710-2715, 2000.

25. Boucher Y, Baxter LT and Jain RK: Interstitial pressure gradients in tissue-isolated and subcutaneous tumors: implications for therapy. Cancer Res 50: 4478-4484, 1990.

26. Minchinton AI and Tannock IF: Drug penetration in solid tumours. Nat Rev Cancer 6: 583-592, 2006

27. Jang SH, Wientjes MG, Lu D and Au JL: Drug delivery and transport to solid tumors. Pharm Res 20: 1337-1350, 2003.

28. Economopoulou M, Bdeir K, Cines DB, et al: Inhibition of pathologic retinal neovascularization by $\alpha$-defensins. Blood 106: 3831-3838, 2005.

29. Zasloff M: Antimicrobial peptides of multicellular organisms. Nature 415: 389-395, 2002

30. McPhee JB and Hancock RE: Function and therapeutic potential of host defence peptides. J Pept Sci 11: 677-687, 2005.

31. Mader JS, Salsman J, Conrad DM and Hoskin DW: Bovine lactoferricin selectively induces apoptosis in human leukemia and carcinoma cell lines. Mol Cancer Ther 4: 612-624, 2005.

32. Moore AJ, Devine DA and Bibby MC: Preliminary experimental anticancer activity of cecropins. Pept Res 7: 265-269, 1994.

33. Jacob L and Zasloff M: Potential therapeutic applications of magainins and other antimicrobial agents of animal origin. Ciba Found Symp 186: 197-216, 1994.

34. Hui L, Leung K and Chen HM: The combined effects of antibacterial peptide cecropin A and anti-cancer agents on leukemia cells. Anticancer Res 22: 2811-2816, 2002.

35. Johnstone SA, Gelmon K, Mayer LD, Hancock RE and Bally MB: In vitro characterization of the anticancer activity of membraneactive cationic peptides. I. Peptide-mediated cytotoxicity and peptide-enhanced cytotoxic activity of doxorubicin against wild-type and P-glycoprotein over-expressing tumor cell lines. Anticancer Drug Des 15: 151-160, 2000.

36. Lincke CR, van der Bliek AM, Schuurhuis GJ, van der VeldeKoerts T, Smit JJ and Borst P: Multidrug resistance phenotype of human BRO melanoma cells transfected with a wild-type human $m d r l$ complementary DNA. Cancer Res 50: 1779-1785, 1990.

37. Ohsaki Y, Gazdar AF, Chen HC and Johnson BE: Antitumor activity of magainin analogues against human lung cancer cell lines. Cancer Res 52: 3534-3538, 1992.

38. Fojo AT and Menefee M: Microtubule targeting agents: basic mechanisms of multidrug resistance (MDR). Semin Oncol 32 (Suppl 7): S3-S8, 2005.

39. Doyle L and Ross DD: Multidrug resistance mediated by the breast cancer resistance protein BCRP (ABCG2). Oncogene 22: 7340-7358, 2003.

40. Gottesman MM, Fojo T and Bates SE: Multidrug resistance in cancer: role of ATP-dependent transporters. Nat Rev Cancer 2: 48-58, 2002. 
41. Xu RH, Pelicano H, Zhou Y, et al: Inhibition of glycolysis in cancer cells: a novel strategy to overcome drug resistance associated with mitochondrial respiratory defect and hypoxia. Cancer Res 65: 613-621, 2005.

42. Winkler F, Kozin SV, Tong RT, et al: Kinetics of vascular normalization by VEGFR2 blockade governs brain tumor response to radiation: role of oxygenation, angiopoietin-1, and matrix metalloproteinases. Cancer Cell 6: 553-563, 2004.

43. Baluk P, Morikawa S, Haskell A, Mancuso M and McDonald DM: Abnormalities of basement membrane on blood vessels and endothelial sprouts in tumors. Am J Pathol 163: 1801-1815, 2003.

44. Morikawa S, Baluk P, Kaidoh T, Haskell A, Jain RK and McDonald DM: Abnormalities in pericytes on blood vessels and endothelial sprouts in tumors. Am J Pathol 160: 985-1000, 2002.
45. Adams RH and Alitalo K: Molecular regulation of angiogenesis and lymphangiogenesis. Nat Rev Mol Cell Biol 8: 464-478, 2007.

46. Goel S, Duda DG, Xu L, et al: Normalization of the vasculature for treatment of cancer and other diseases. Physiol Rev 91: 1071-1121, 2011.

47. Huang Y, Yuan J, Righi E, et al: Vascular normalizing doses of antiangiogenic treatment reprogram the immunosuppressive tumor microenvironment and enhance immunotherapy. Proc Natl Acad Sci USA 109: 17561-17566, 2012. 